\title{
Stress Corrosion Crack Growth Mechanism on SUS316L Stainless Steel
}

\author{
Hiroyuki Masuda*
}

\author{
Intense Research Group, National Institute for Material Science, 1-2-1 Sengen, Tsukuba, Ibaraki, 305-0047, Japan
}

\begin{abstract}
The stress corrosion cracking (SCC) of stainless steel is one of the biggest problems for maintaining atomic power and chemical plants. However the mechanism has not been solved because of difficulty in observing hydrogen movement. In order to solve this problem, the author has developed a new SCC test method that enables the super Kelvin force microscope (SKFM) and the Kelvin force microscope (KFM) observations. By using this test method, the crack tip deformation and surface potential distribution on SUS316L stainless steels were observed by SKFM and KFM. The existence of hydrogen-induced martensite was examined by the magnetic force microscope (MFM) observations. The results showed that a less noble potential region existed near the crack tip. MFM and KFM observation showed hydrogeninduced martensite existed at the less noble potential region. Repeated SKFM observations revealed that the crack is formed by the movement of hydrogen-induced martensite.
\end{abstract}

\section{INTRODUCTION}

The stress corrosion cracking (SCC) of stainless steel is one of the biggest problems that have not been solved. Many excellent research works [1-8] have been done from the aspects of mechanical factors (applied stress, residual strain), environmental factors (temperature, $\mathrm{pH}$, dissolved oxygen content) and material factors (additional element, structure) to clarify the mechanism of SCC. Three basic mechanisms of SCC have been proposed, such as active path dissolution, hydrogen embrittlement and film induced cleavage. However, no clear mechanism of SCC has been found in the above-mentioned environment. The author has developed a new SCC test device [9] that enables the super Kelvin force microscope (SKFM) [10] observation. By using this test device, surface potential distribution was observed on the whole crack of SUS304 and SUS310S [11] stainless steel. It has been found that less noble potential region existed near the crack tip on SUS304 stainless steel and around the crack on SUS310S stainless steel. Ag decoration [12] was done at the same time to study the relation between the less noble potential region and hydrogen distribution. The results showed that there is a good correlation between them [11, 13]. The magnetic force microscope (MFM) and the Kelvin force microscope (KFM) observations at the less noble potential region on SUS310S stainless steel revealed that hydrogen-induced martensite existed at the less noble potential region. SUS316L stainless steel is austenitic stainless steel that is commonly used at atomic power plant. To clarify the mechanism of SCC on SUS316L stainless steel, the crack morphology and surface potential distribution were observed by SKFM and KFM. At the same time, the distribution of hydrogen was examined by the $\mathrm{Ag}$ decoration method with the energy dispersed X-ray (EDX) image mapping analysis combined. The existence of hydrogeninduced martensite was examined by MFM observation.

*Address correspondence to this author at the Intense Research Group, National Institute for Material Science, 1-2-1 Sengen, Tsukuba, Ibaraki, 305-0047, Japan; Tel: 0081-029-859-2542; Fax: 0081-029-859-2501;

E-mail: masuda.hiroyuki@nims.go.jp
Moreover, the crack growth behavior was studied by repeating SKFM observation. The mechanism of SCC is discussed from the results of these observations.

\section{MATERIAL AND METHODS}

\section{SPM Equipment}

SKFM was originally developed by the author. The Kelvin force method used in this device was originally developed by Yasutake et al. [14]. In this method, topography and surface potential can be obtained at the same time with non-contact mode. The scanning device of SKFM for $X-Y$ direction used is an accurate $X-Y$ stage. The accuracy of the $X-Y$ stage is less than $100 \mathrm{~nm}$ for repeated positioning. The $X-Y$ stage can move up to $10 \times 10 \mathrm{~cm}^{2}$, but the maximum scanning area is limited to $1 \times 1 \mathrm{~cm}^{2}$. The minimum vertical movement is $0.2 \mathrm{~mm}$. KFM and MFM observation were done by SPI8000 (SII). The tip used for KFM and SKFM measurement was the conductive goldcoated Si tip with the resonant frequency of around $25 \mathrm{kHz}$, while the tip used for MFM measurement was CoPtCrcoated Si tip with the resonant frequency of around $125 \mathrm{kHz}$. The SKFM image was taken with a scanning speed of between 0.03 and $0.06 \mathrm{~Hz}$ with data points of $256 \times 256$, while KFM and MFM image were taken with a scanning speed of $0.1 \mathrm{~Hz}$ with data points of $256 \times 256$. The bright part corresponds to less noble potential part in SKFM and KFM image, while the bright part corresponds to the part where attractive force is operating (martensite part) in MFM image.

\section{SCC Test}

Test specimens used were commercial SUS316L stainless steel (Cr: 17.37, Ni: 12.11, Mo: 2.05, C: 0.014, Si: 0.58 , Mn 1.54, P: 0.031 , S: 0.002 ) with the size of $40 \mathrm{~mm}$ to $100 \mathrm{~mm}$ in length, $20 \mathrm{~mm}$ in width, $0.2 \mathrm{~mm}$ (Vickers hardness (HV): $310,50 \mathrm{gf})$ and $0.1 \mathrm{~mm}$ (HV: 190, 50gf) in thickness. The test part of specimen was mechanically polished up to $300 \mathrm{~nm}$ in roughness. Stress was applied by bending the specimen with a jig of $40 \mathrm{~mm}$ to $80 \mathrm{~mm}$ in 
length. The stress distribution of specimen, F, was estimated from the following formula:

$\mathrm{F}=\mathrm{z} * \mathrm{E} / \mathrm{R}$

where $\mathrm{z}$ is distance from the center part of thickness of specimen, E is Young's modulus and $\mathrm{R}$ is curvature of radius of the test specimen. Thus the tensile stress is maximum at the surface and zero at the center part of thickness of the specimen. More than 10 pieces of $25 \% \mathrm{MgCl}_{2}$ droplet of 2 $\mathrm{mm}^{3}$ in volume were attached on the middle part of the specimen [9]. Test was done at $343 \mathrm{~K}$ and $28 \% \mathrm{RH}$. The specimen was washed by water after the test. At first, the crack was observed by the color laser microscope and then observed by SKFM or by both KFM and MFM. After SKFM observation, the specimen was immersed in $0.043 \mathrm{M} / \mathrm{L}$ $\mathrm{AgK}(\mathrm{CN})_{2}$ solution about $14 \mathrm{~h}$ for $\mathrm{Ag}$ decoration. In $\mathrm{Ag}$ decoration method, Ag precipitation occurs on the part where absorbed hydrogen exists as the following chemical reaction: $\mathrm{H}_{\text {abs }}$ becomes $\mathrm{H}^{+}$and $\mathrm{Ag}^{+}$becomes $\mathrm{Ag}$.

After specimen was washed by water and dried, EDX image mapping analysis was carried out around the crack following the color laser microscope observation.

\section{Crack Growth Test}

The following (a) to (d) were repeated for the crack growth test; (a) The specimen with more than 10 pieces of $\mathrm{MgCl}_{2}$ droplets attached was kept at $343 \mathrm{~K}$ and $28 \% \mathrm{RH}$ with maximum applied stress of $1080 \mathrm{MPa}$ for 1 day. (b) The specimen was washed by water and dried and observed by the color laser microscope. (c) SKFM observation was done at $298 \mathrm{~K}$ and below $20 \% \mathrm{RH}$ for 2 days. (d) $\mathrm{MgCl}_{2}$ droplets were attached again on the specimen nearly the same place as before.

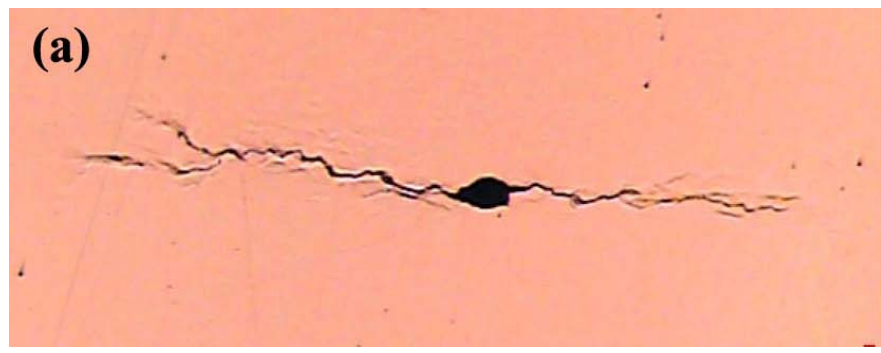

(b)

Fig. (1). Optical microscope images of crack produced at applied stress of $1030 \mathrm{MPa}$ for 8 days. (a) Before Ag decoration, (b) after Ag decoration.
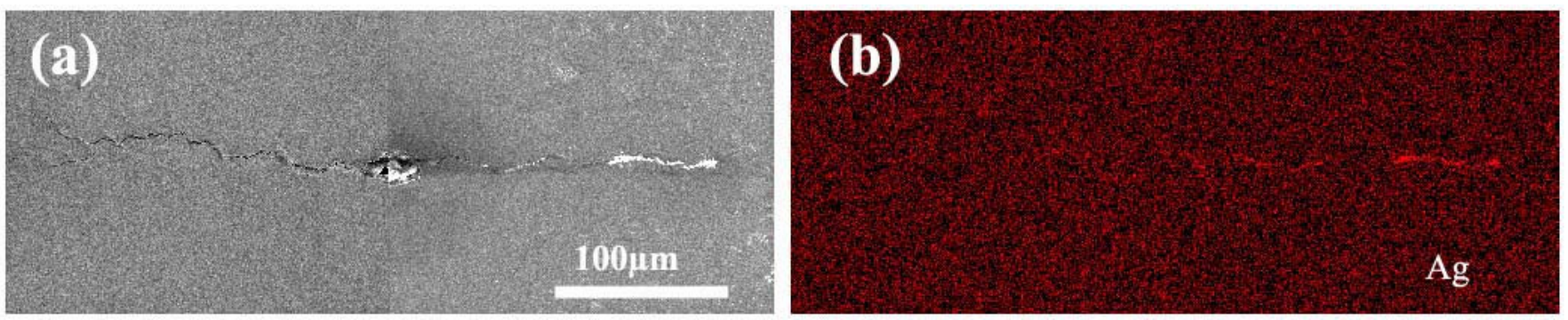

Fig. (2). EDAX image mapping analysis of crack produced at applied stress of $1030 \mathrm{MPa}$ for 8 days. (a) SEM image, (b) EDX image mapping of Ag.
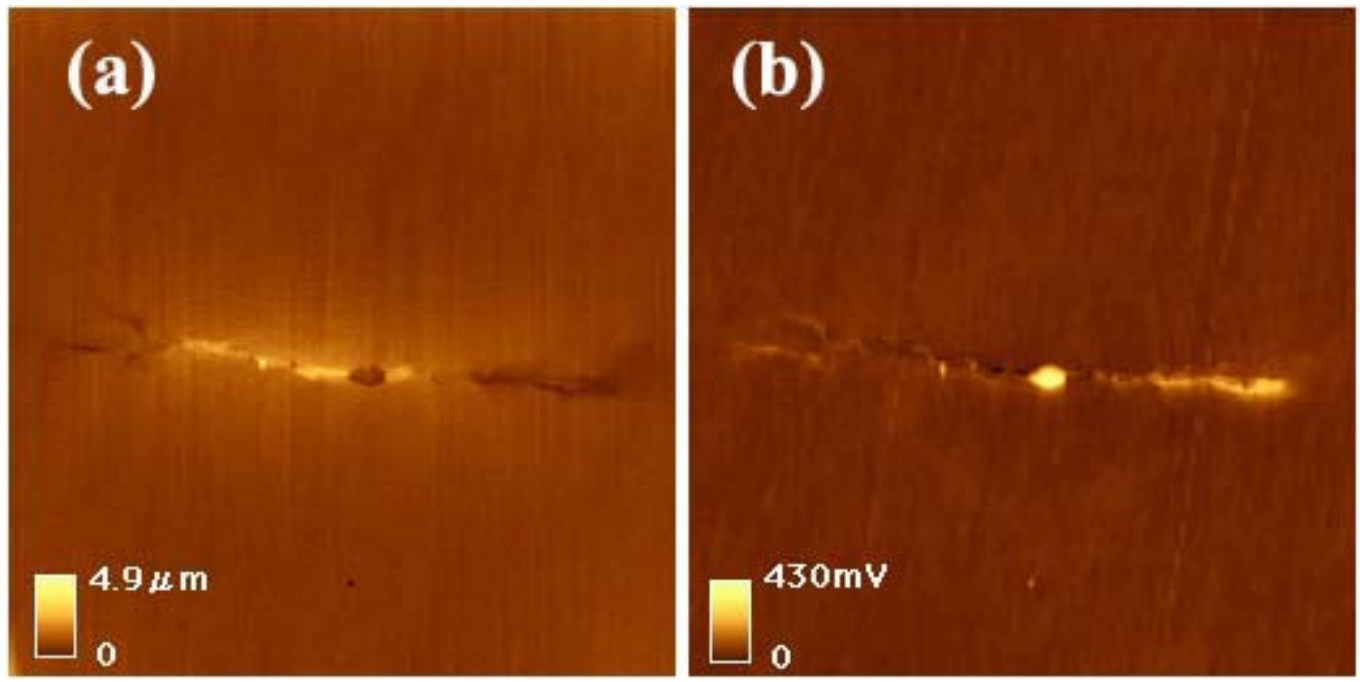

Fig. (3). SKFM image of crack produced at applied stress of $1030 \mathrm{MPa}$ for 8 days. Image size: $0.6 \mathrm{~mm} \mathrm{X} 0.6 \mathrm{~mm}$. (a) Topography, (b) potential distribution image. 


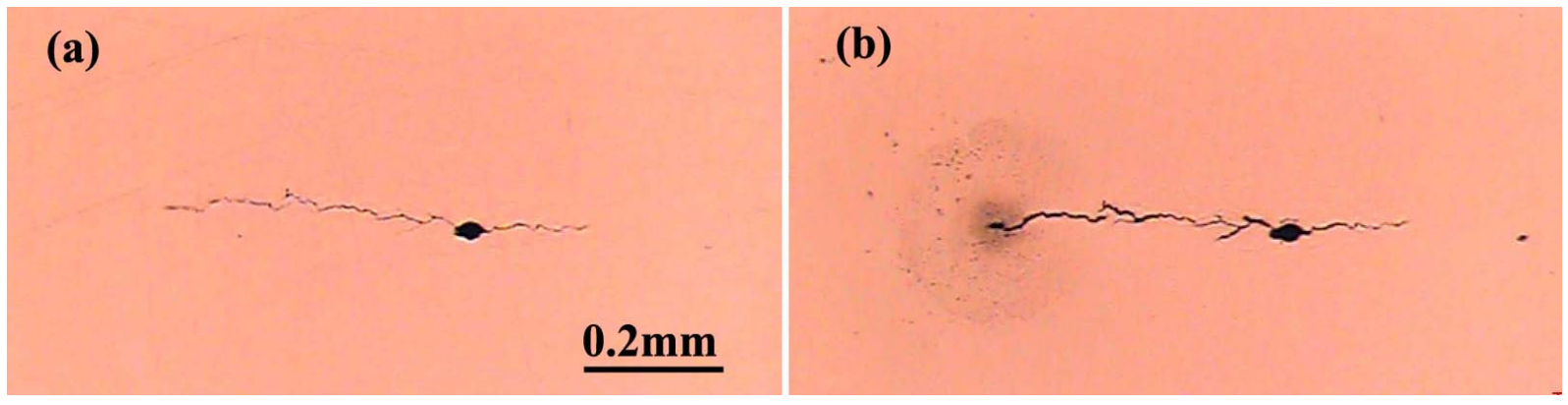

Fig. (4). Optical microscope images of crack produced at applied stress of $588 \mathrm{MPa}$ for 8 days. (a) Before Ag decoration, (b) after Ag decoration.
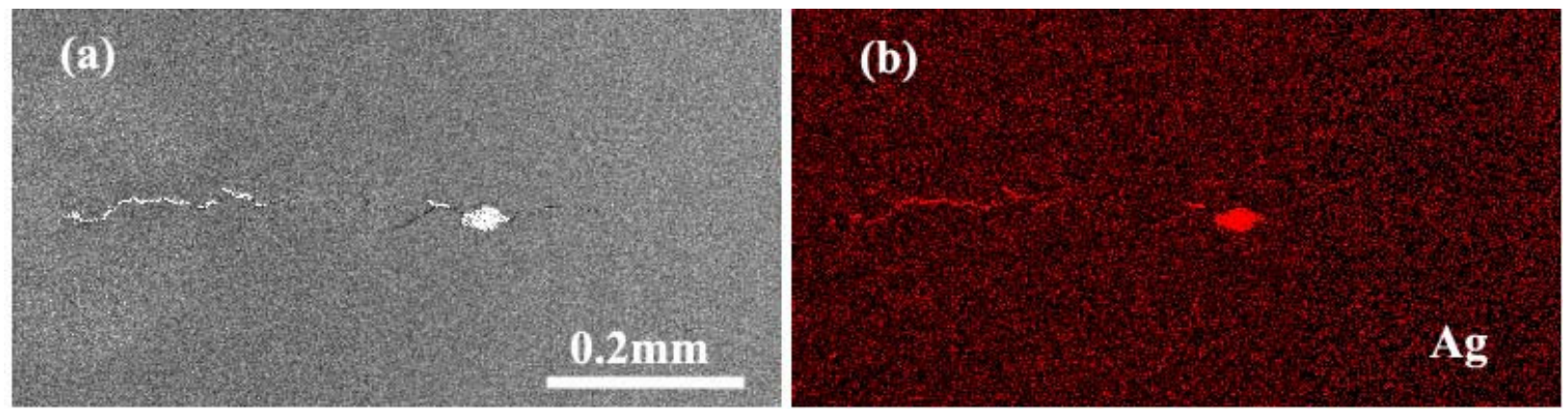

Fig. (5). EDAX image mapping analysis of crack produced at applied stress of $588 \mathrm{MPa}$ for 8 days. (a) SEM image, (b) EDX image mapping of Ag.
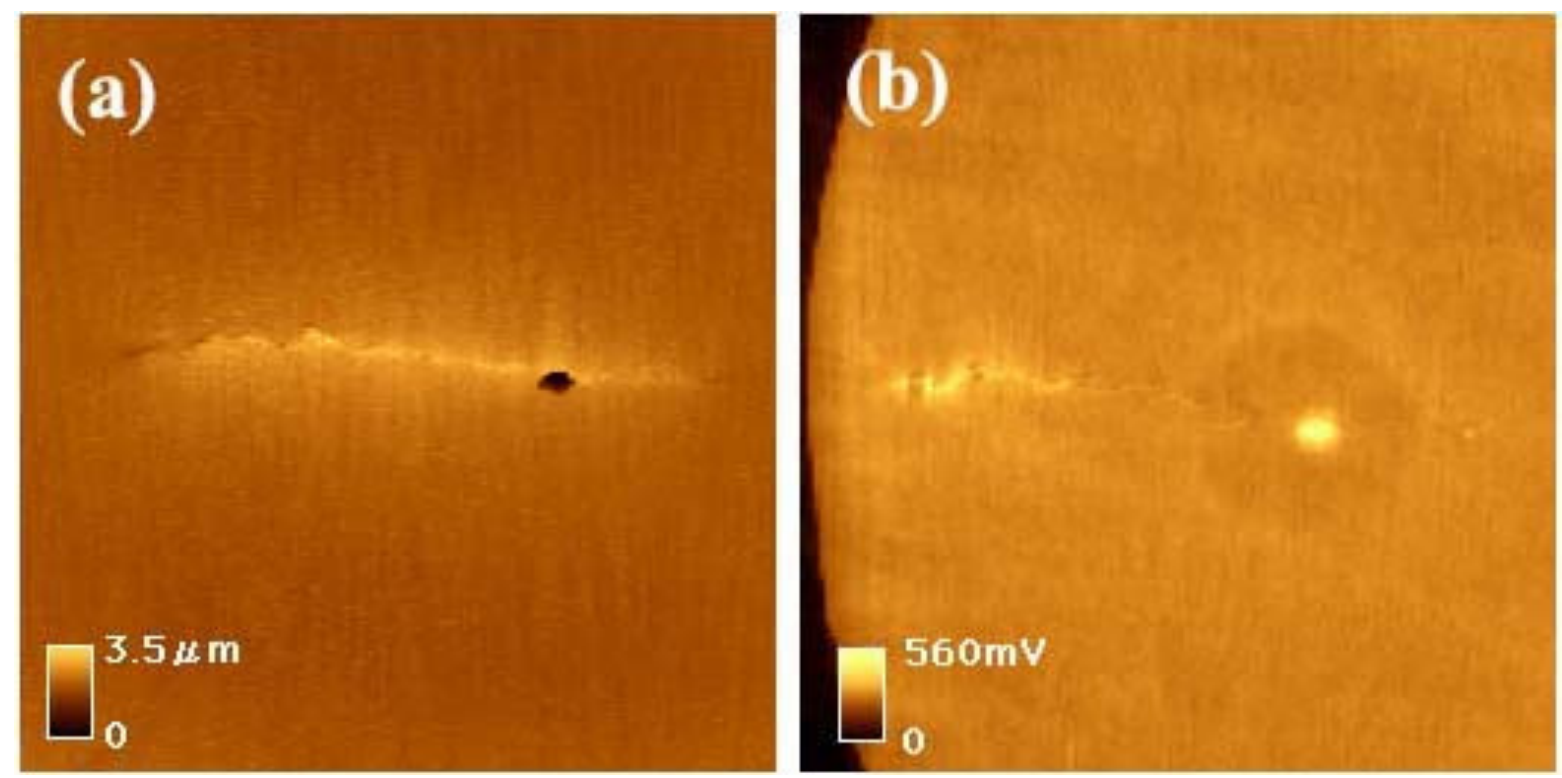

Fig. (6). SKFM image of crack produced at applied stress of $588 \mathrm{MPa}$ for 8 days. Image size: $0.6 \mathrm{~mm} \mathrm{X} 0.6 \mathrm{~mm}$. (a) Topography, (b) potential distribution image.

\section{RESULT AND DISCUSSION}

\section{HV: 310}

Fig. (1) shows the optical microscope images of crack (a) before Ag decoration and (b) after Ag decoration. The crack was produced at applied stress of $1030 \mathrm{MPa}$ for 8 days. The crack usually initiated from a pit and propagated to the maximum stress direction (horizontal direction). Ag which is proved by EDX image mapping analysis (Fig. 2) precipitated only right side of the crack tip. The change of color was often observed near the crack tip where large amount of silver precipitated. Fig. (3) shows the SKFM observation of the crack. Less noble potential region was observed near the crack tip of right side. Fig. (4) shows the optical microscope images of crack (a) before Ag decoration and (b) after Ag decoration. The crack was produced at applied stress of 588 $\mathrm{MPa}$ for 8 days. Ag which is proved by EDX image mapping analysis (Fig. 5) precipitated in the pit and left side of the crack tip. The precipitation of $\mathrm{Ag}$ in the pit was usually observed in this stress level. The change of color was observed at the left side of the crack tip. Fig. (6) shows SKFM observation of the crack. Less noble potential region was observed near the crack tip of left side. Thus there is a very good correlation between hydrogen distribution and less 

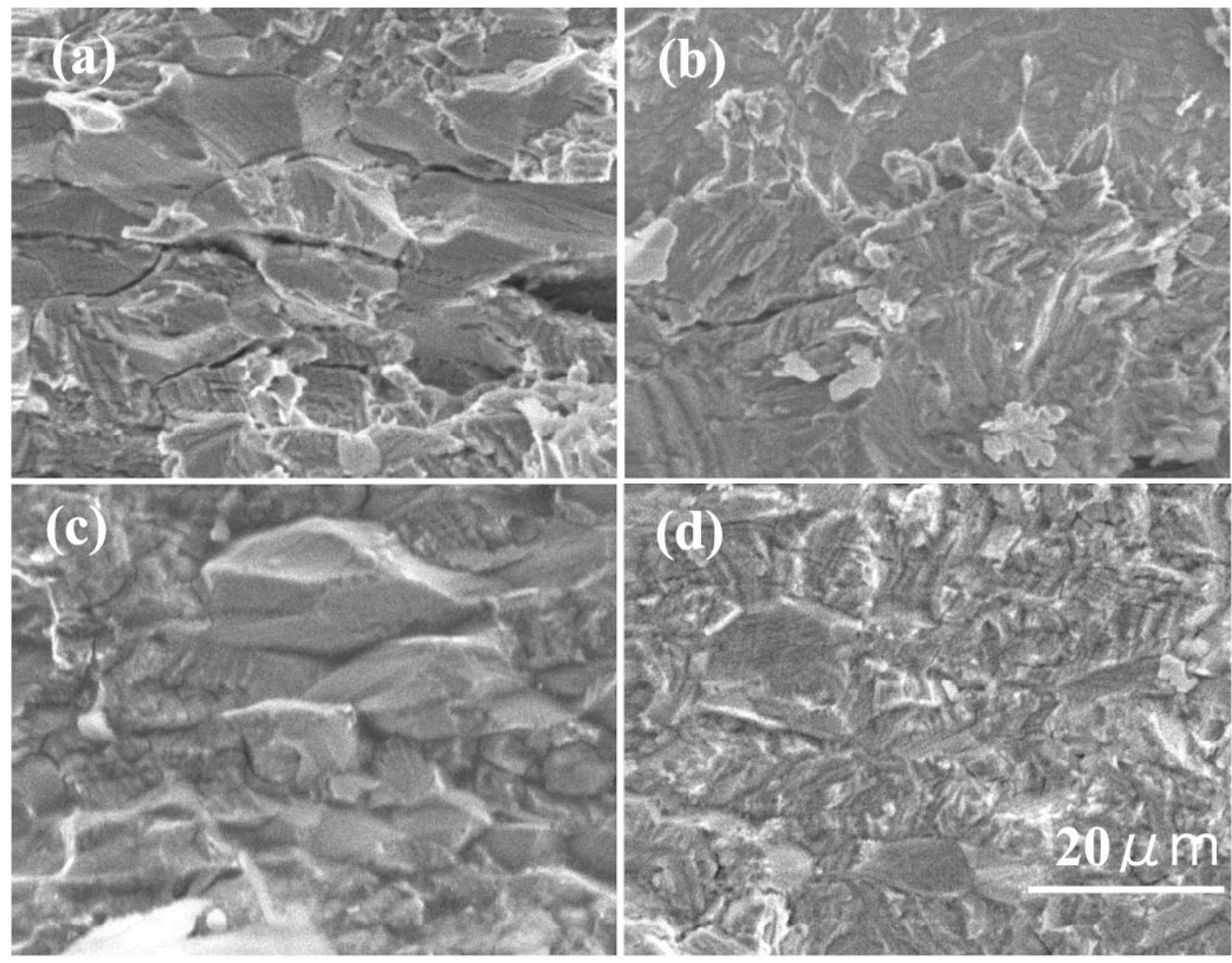

Fig. (7). SEM images of fracture surface produced at applied stress of (a), (b) $1030 \mathrm{MPa}$ for 30 days and (c), (d) $588 \mathrm{MPa}$ for 33 days.

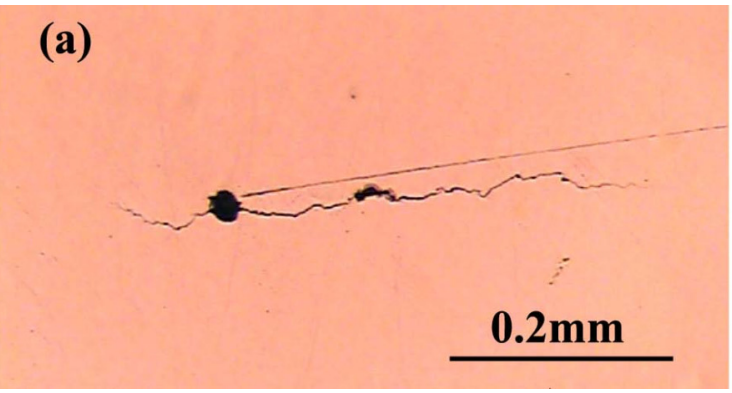

(b)

Fig. (8). Optical microscope images of crack produced at applied stress of $515 \mathrm{MPa}$ for 12 days. (a) Before Ag decoration, (b) after Ag decoration.

noble potential region. Fig. (7) shows SEM images of the fracture surface produced at applied stress of (a), (b) 1030 $\mathrm{MPa}$ for 30 days and (c), (d) $588 \mathrm{MPa}$ for 33 days. Intergranular and transgranular fracture surfaces were observed both on the fracture surface produced at applied stress of $1030 \mathrm{MPa}$ and $588 \mathrm{MPa}$. No difference was found on the fracture surface produced at applied stress of 1030 $\mathrm{MPa}$ and $588 \mathrm{MPa}$.

\section{HV: 190}

Fig. (8) shows the optical microscope images of crack (a) before $\mathrm{Ag}$ decoration and (b) after Ag decoration. The crack was produced at the applied stress of $515 \mathrm{MPa}$ for 12 days. The crack usually initiated from a pit and propagated to the maximum stress direction (horizontal direction). Ag which is proved by EDX image mapping analysis (Fig. 9) precipitated only right side of the crack. Fig. (10) shows SKFM observation of the crack. Less noble potential region was observed near the crack tip of right side. There is very good correlation between the less noble potential region and the region of Ag precipitation. Thus less noble potential region is closely related to the hydrogen distribution. The effect of hardness on SCC behavior is that the ratio of crack initiation probability is different. That is, cracks initiated from most droplets when HV is 310 , while cracks initiated from few droplets when HV is 190.

\section{MFM and KFM Observation}

Fig. (11) shows the optical microscope images of (a) whole crack and crack tip surrounded by square line (b) at lower part and (c) at upper part in Fig. (11a, d) topography of Fig. (11b, e) potential distribution image, (f) magnetic 

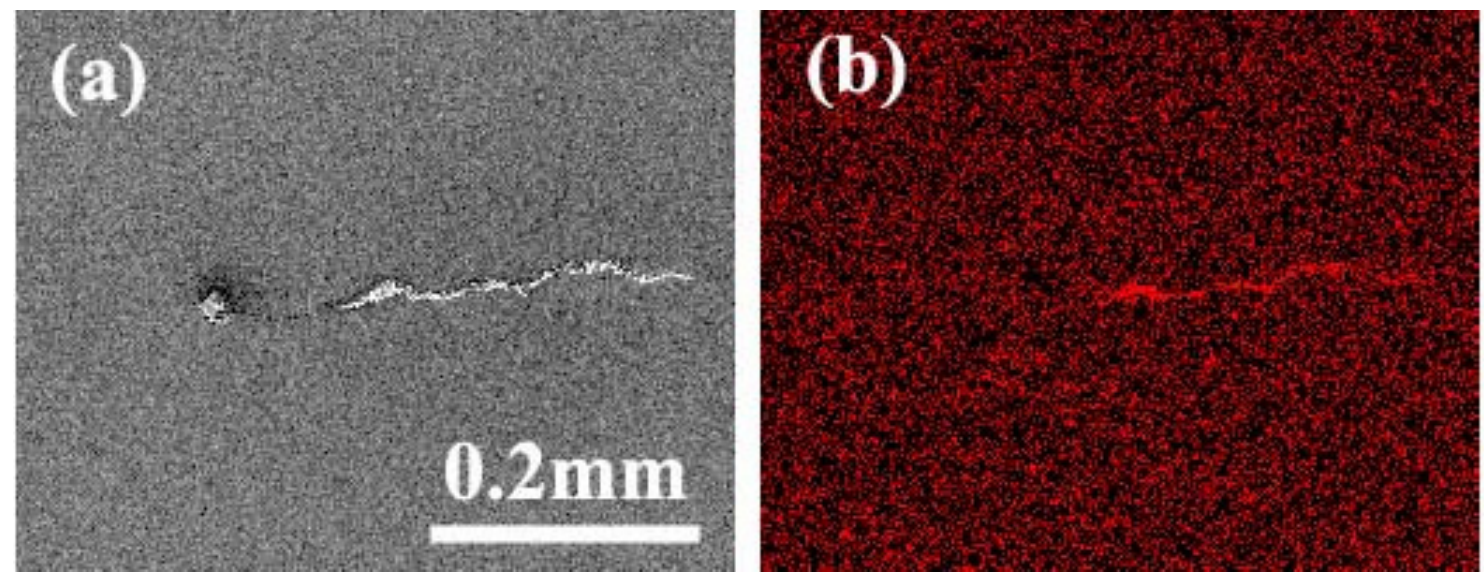

Fig. (9). EDAX image mapping analysis of crack produced at applied stress of $512 \mathrm{MPa}$ for 12 days. (a) SEM image, (b) EDX image mapping of Ag.

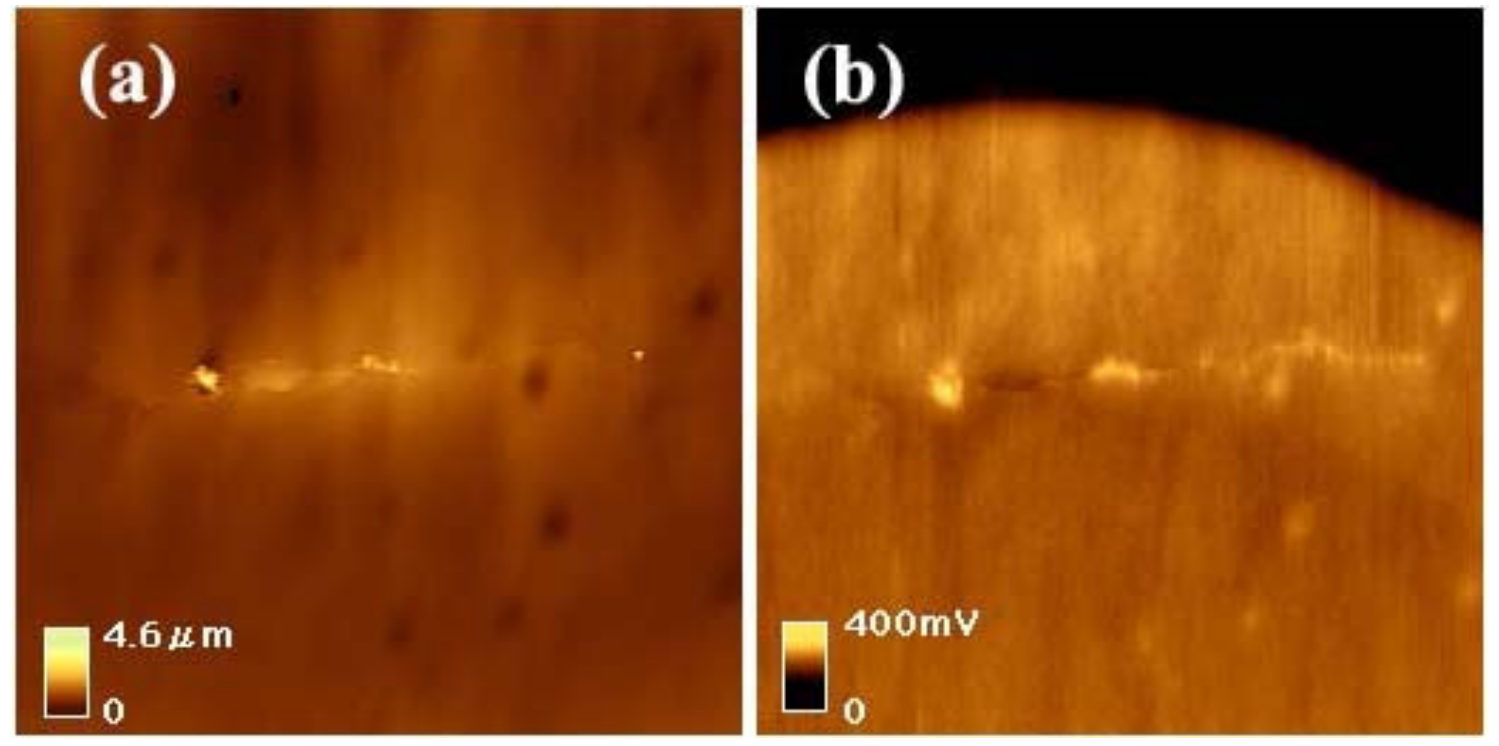

Fig. (10). SKFM images of crack produced at applied stress of $512 \mathrm{MPa}$ for 12 days. Image size: $0.6 \mathrm{~mm}$ X $0.6 \mathrm{~mm}$. (a) Topography, (b) potential distribution image.

force distribution image, (g) topography of Fig. (11c, h) potential distribution image and (i) magnetic force distribution image. The crack was produced at applied stress of $1030 \mathrm{MPa}$ for 4 days. It is clear from the optical microscope image of Fig. (11b, c) that the crack propagates discontinuously. Topography (Fig. 11d) shows that plastic deformation occurs ahead of the crack tip. Less noble potential region was observed around the crack tip in Fig. (11e) and only part of crack in Fig. (11h). MFM observation shows that the less noble potential region corresponds to the region where the attractive force exists. Since the attractive magnetic force does not generate on austenite phase, martensite phase must exist. This martensite is considered hydrogen-induced martensite, since there is no correlation between plastic deformation and martensite phase. The area of martensite phase shown in Fig. (11i) is less than that shown in Fig. (11f). The difference of the area of martensite phase might affect the activity of the crack growth.

\section{Crack Growth Test}

Fig. (12) shows SKFM images of whole crack and optical microscope images surrounded by square line in
SKFM images. The optical microscope images shown in Fig. (12) are the same place. Less noble potential region was observed ahead of the crack tip where plastic deformation occurs (Fig. 12b) after 2 days loading test. Crack was not formed at this moment (Fig. 12c). Less noble potential region moved as a new plastic deformation occurred (Fig. 12e) after 3 days loading test. Then crack was formed (Fig. 12f). The less noble potential region corresponds to the hydrogen-induced martensite phase as shown in Fig. (11), so the movement of less noble potential region indicates the movement of hydrogen. Thus crack was formed when hydrogen moved.

\section{Mechanism of SCC}

Narita et al. [15] studied hydrogen-related phase transformations in austenitic stainless steel by using X-ray diffraction technique and reported that volume increase occurs and compressive stress generates when hydrogen is charged on austenitic stainless steel, and tensile stress generates when charged hydrogen is lost by aging. From repeated SKFM observations as shown in Fig. (12), it is clear that hydrogen tends to accumulate in the plastic deformation 

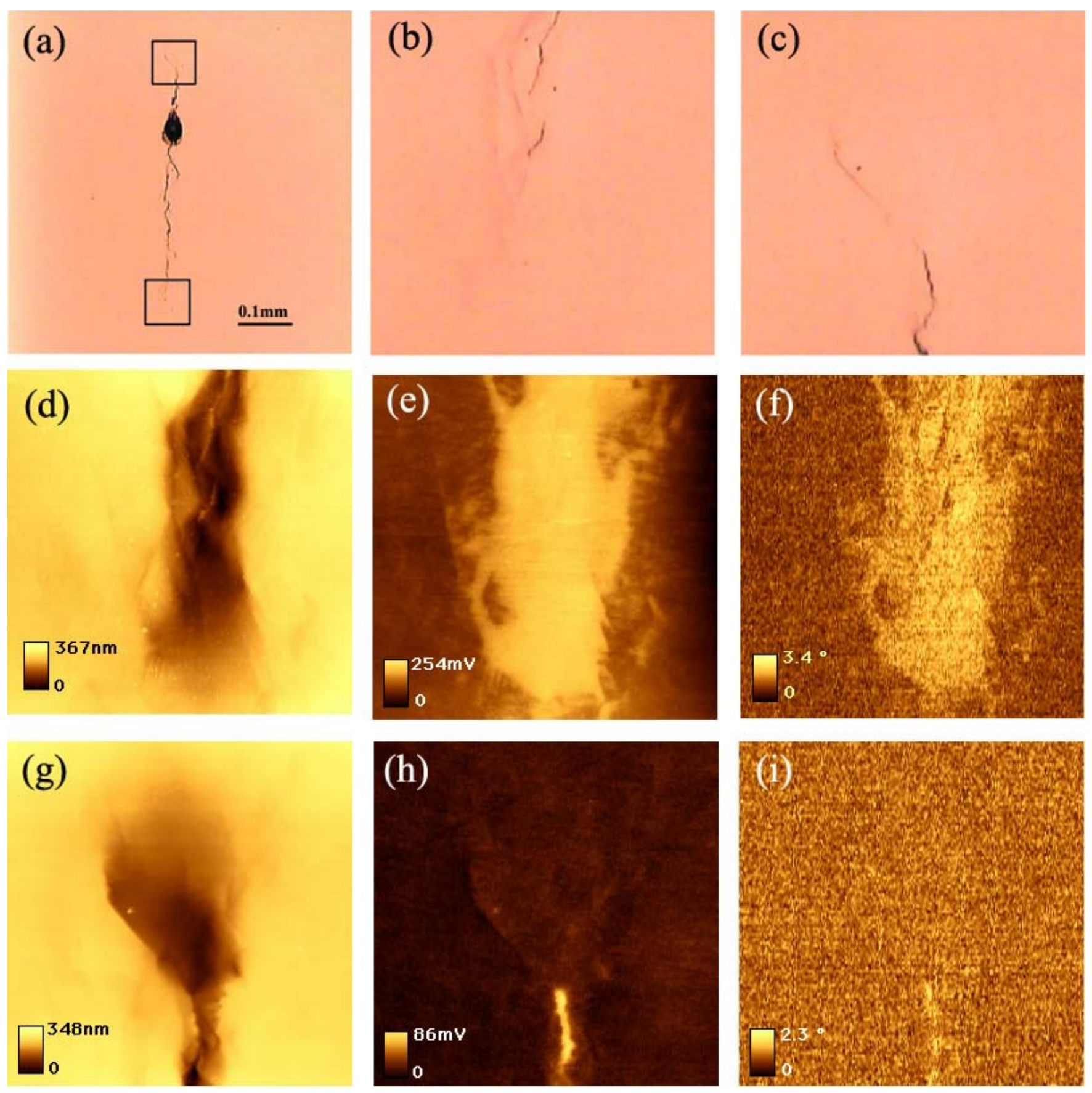

Fig. (11). Images of crack produced at applied stress of $1030 \mathrm{MPa}$ for 4 days. Image size except (a): $0.08 \mathrm{~mm} \mathrm{X} 0.08 \mathrm{~mm}$. Optical microscope image of (a) whole crack and crack tip surrounded by square line (b) at lower part and (c) at upper part in Fig. (11a, d) topography of Fig. (11b, e) potential distribution image, (f) magnetic force distribution image, (g) topography of Fig. (11c, h) potential distribution image, (i) magnetic force distribution image.

region ahead of the crack tip. This accumulation of hydrogen is considered to generate compressive stress. As the result, new plastic deformation occurs when the accumulated hydrogen reaches to certain amount. Then hydrogen moves to the newly created plastic deformation region and tensile stress generates at the region where hydrogen-induced martensite phase changes to austenite phase. The tensile stress increases with the movement of hydrogen to cause crack. Thus the crack growth is caused by the hydrogen movement under loading condition.

\section{CONCLUSION}

The crack tip deformation and surface potential distribution were observed by SKFM and KFM. At the same time, the distribution of hydrogen was examined by the $\mathrm{Ag}$ decoration method with the EDX image mapping analysis combined. Moreover, the existence of hydrogen-induced martensite was examined by MFM. The results showed that less noble potential region existed near the crack tip and $\mathrm{Ag}$ precipitated at the less noble potential region in most cases. MFM and KFM observation showed hydrogen-induced 


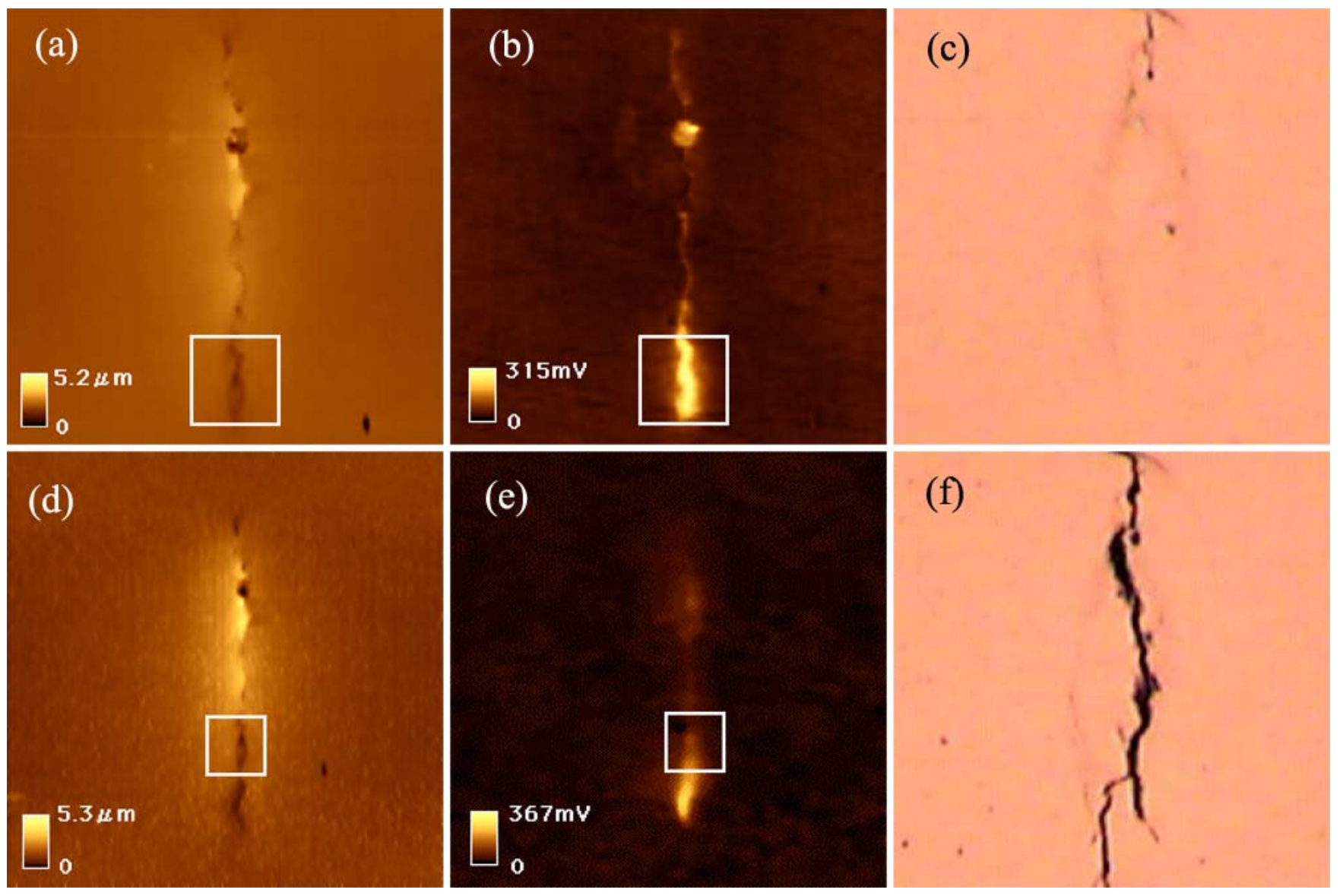

Fig. (12). SKFM images and optical microscope images surrounded by square line in SKFM images. (a) Topography, (b) surface potential distribution image, (c) optical microscope image after 2 days test and (d) topography, (e) surface potential image, (f) optical microscope image after 3 days test. Image size: (a), (b) $0.4 \mathrm{~mm} \mathrm{X} 0.4 \mathrm{~mm}$, (c) $0.08 \mathrm{~mm} \mathrm{X} 0.08 \mathrm{~mm}$, (d), (e) $0.6 \mathrm{~mm} \mathrm{X} 0.6 \mathrm{~mm}$, (f) $0.08 \mathrm{~mm} \mathrm{X} 0.08 \mathrm{~mm}$.

martensite existed at the less noble potential region. Crack is produced in the process that hydrogen-induced martensite phase becomes former austenite phase.

\section{REFERENCES}

[1] Hoar TP, Hines JG. The stress corrosion cracking of austenitic stainless steel. J Iron Steel Inst 1956; 182: 124-43.

[2] Hoar TP, Scully JC. Mechanism of anodic dissolution of austenitic stainless steel in hot chloride solution of controlled electrode potential. J Electrochem Soc 1964; 111: 348-52.

[3] Uhlig $\mathrm{HH}$, Cook EV. Mechanism of inhibiting stress corrosion cracking of 18-8 stainless steek in $\mathrm{MgCl}_{2}$ by acetates and nitrates. $\mathrm{J}$ Electrochem Soc 1969; 116: 173-7.

[4] Nielsen NA. Observation and thoughts on stress corrosion mechanisms. Corrosion 1971; 27: 173-89.

[5] Whiteman MB, Troiano AR. Hydrogen embrittlement of austenitic stainless steel. Corrosion 1965; 21: 53-6.

[6] Holzworth ML. Hydrogen embrittlement of Type 304L stainless steel. Corrosion 1969; 25: 107-15.

[7] Vaughan DA, Phalen DI, Peterson CL, Boyd WK. Relationship between hydrogen pickup and susceptible paths in stress corrosion cracking of Type 304 stainless steels. Corrosion 1963; 19: 315t$326 \mathrm{t}$.

[8] Rhodes P. Mechanism of chloride stress corrosion cracking of austenitic stainless steels. Corrosion 1969; 25: 462-72.

[9] Masuda H. SKFM observation of SCC on SUS304 stainless steel. Corrosion Sci 2007; 49: 120-9.

[10] Masuda H. Effect of $\mathrm{MgCl}_{2}$ liquid thickness on atmospheric corrosion of pure iron. Corrosion 2001; 57: 99-109.

[11] Masuda H. Mechanism of SCC on SUS310S stainless steel. Open Corrosion J 2008; 1: 6-11.

[12] Schober T, Dieker C. Observation of local hydrogen on nickel surfaces. Metall Trans 1983; 14A: 2440-2.

[13] Masuda H. Effect of stress on hydrogen distribution around SCC crack of SUS304 stainless steel. ECS Transaction 2008; 11(22): 5770 .

[14] Yasutake M, Aoki D, Fujihira M. Surface potential measurements using the Kelvin probe force microscope. Thin Solid Films 1996; 273: 279-83.

[15] Narita N, Altstetter CJ, Birnbaum HK. Hydrogen-related phase transformations in austenitic stainless steels. Metall Trans 1982; 13A: $1355-65$.

This is an open access article licensed under the terms of the Creative Commons Attribution Non-Commercial License (http://creativecommons.org/licenses/by$\mathrm{nc} / 3.0 /$ ) which permits unrestricted, non-commercial use, distribution and reproduction in any medium, provided the work is properly cited. 